\title{
La réflexivité : Une question unique, des approches et des phénomènes différents
}

\section{Gian Maria Tore}

\section{(2) OpenEdition}

\section{Journals}

Édition électronique

URL : http://journals.openedition.org/signata/548

DOI : $10.4000 /$ signata. 548

ISSN : 2565-7097

Éditeur

Presses universitaires de Liège (PULg)

\section{Édition imprimée}

Date de publication : 31 décembre 2013

Pagination : 53-83

ISBN : 978-2-87562-042-2

ISSN : 2032-9806

\section{Référence électronique}

Gian Maria Tore, "La réflexivité : Une question unique, des approches et des phénomènes différents », Signata [En ligne], 4 | 2013, mis en ligne le 30 septembre 2016, consulté le 02 mai 2019. URL : http:// journals.openedition.org/signata/548 ; DOI : 10.4000/signata.548 


\section{CRITIQUE DU MÉTALANGAGE}

\section{La réflexivité : \\ Une question unique, des approches et des phénomènes différents ${ }^{1}$}

Gian Maria Tore

Université du Luxembourg

\section{Introduction : la réflexivité comme question fondamentale mais marginale des sciences humaines}

La réflexivité: une question qui traverse de bout en bout, latente ou flagrante, le vaste et multiforme continent des sciences humaines. De la philosophie à la psychologie, de la logique à la linguistique, de la sémiotique à la théorie de la littérature et de l'art, de l'anthropologie à la théorie du droit... pratiquement n'importe quelle discipline qui étudie le sens traite la réflexivité comme un objet théorique essentiel, tout en l'abordant et même le nommant différemment. J'aimerais dire : tout en le perdant de vue, précisément au travers d'une myriade d'approches et de dénominations. Car à bien voir, les sciences du langage, par exemple, traitent de phénomènes réflexifs dans ce qu'on appelle, selon les auteurs et les approches : sui-référence, opacité énonciative, attitudes propositionnelles, modalisation, monstration, modélisation interne, métalangage, métadiscours, etc. Dans les études littéraires, mais aussi les études de cinéma et d'arts plastiques, il est question de réflexivité par d'autres dénominations, dont la liste complète pourrait être très longue; en voici les plus courantes : métalittérature et métafiction (avec métathéâtre et métacinéma), métarécit, autoreprésentation, spécularité et mise en abyme $^{2}$; et encore :

1. Je voudrais remercier ici tous ceux qui ont écouté ou lu des versions précédentes de cette étude et qui, avec leurs remarques et objections, ont contribué à la qualité (toute relative et provisoire) de cette version; et en particulier : Jean-Marie Klinkenberg et Bernard Vouilloux.

2. Pour les termes littéraires invoqués jusqu'ici, et pour en rallonger la liste, on peut se rapporter aux cartographies de Chanady (1988) et de Weissler (2009) : la première est utile pour notre 
transtexualité ou littérature, art au second degré (avec différentes formes d'intertextualité : citations, plagiats et allusions; et d'hypertextualité : parodies, travestissements, pastiches); et finalement : paratextualité et métatextualité ${ }^{3}$. Face à de telles listes vertigineuses, il est légitime de se demander si, parfois, on ne parle pas essentiellement du même phénomène avec des termes différents et si, d'autres fois, on ne parle pas de phénomènes différents avec un même terme. Ainsi, dans les sciences sociales, où la terminologie est bien plus sobre, on semble se limiter surtout à : réflexivité et autoréférence ${ }^{4}$; mais lorsqu’en sociologie ou en anthropologie on utilise le premier de ces termes, il est question tantôt de la réflexivité de l'observateur, tantôt de la réflexivité de l'acteur ${ }^{5}$.

Le but de cette étude sera non pas de passer en revue les terminologies des différentes disciplines et sous-disciplines ${ }^{6}$, mais d'illustrer l'intérêt qu'il y a à rapprocher les questionnements sur la réflexivité, si disparates puissent-ils sembler. Je voudrais soutenir que la récurrence de tels questionnements n'est nullement anodine et que, si on essaie de la thématiser, on gagne, en retour, une compréhension plus claire et aiguë des phénomènes réflexifs. J'avancerai donc en deux temps : premièrement, en essayant d'embrasser la réflexivité en général, ou encore mieux en essayant d'en faire une question générale - la question générale des sciences humaines peut-être; deuxièmement, à partir de la conception d'une réflexivité généralisée, en distinguant les différentes sortes de phénomènes réflexifs - trop souvent confondus entre eux, identifiés d'une manière contradictoire ou redondante.

propos à cause de son intérêt vers la terminologie «méta (que nous allons discuter par la suite), la seconde est recommandable parce qu'elle est, à notre connaissance, la plus récente et, sans doute, l'une des rares études systématiques de la réflexivité.

3. Pour la transtextualité ou « littérature au second degré », je me limite à renvoyer au panorama raisonné et bien connu de Genette (1982, cf. notamment la présentation pp. 7-19); et pour la paratextextualité et métatextualité, à l'autre panorama de Genette (1987). Par ailleurs, il est intéressant de noter que ces études de Genette, bien qu'elles essaient d'analyser précisément la « littérarité » de la littérature ou la « textualité » des textes, bien qu'elles portent donc explicitement sur la réflexivité, et qu'elles soient fort connues, ne jouent pas un rôle important dans les autres études générales sur la réflexivité littéraire que je viens de citer. C'est là, à mon sens, le signe d'une vue trop parcellisée sur la question de la réflexivité.

4. Je me borne à signaler ici les points de départ majeurs, dans les sciences sociales : pour l'étude de la réflexivité, l'ethnométhodologie (Garfinkel, 1967); pour l'étude de l'autoréférence, la théorie des systèmes (Luhmann, notamment 1984, chap. XI).

5. Tantôt on thématise le fait que l'observateur s'implique dans la pratique sociale qu'il observe (cf. par exemple l'anthropologie « interprétative » de Geertz, 1973 et 1983); tantôt, que l'acteur se dés-implique de sa pratique pour l'observer (cf. l'analyse de la construction et manipulation du «frame» dans Goffman, 1974, sur lequel nous reviendrons; cf. plus simplement, le regard sur la manière qu'une institution a de questionner sa manière de se comporter, sa "réflexivité institutionnelle », dans Goffman, 1977).

6. On sera amené à négliger notamment la philosophie logique. Pour un panorama récent qui ouvre un dialogue entre logique et esthétique sur la réflexivité : Genin (1998). 
Si la tâche ainsi définie peut paraître décourageante, il faut constater que, par excès de prudence (ou par obligation disciplinaire?), rarissimes sont les études qui ont ouvert la question de la réflexivité de manière générale. S'il est courant d'invoquer la réflexivité, il est exceptionnel de l'aborder d'une manière frontale et systématique : on l'assume (à tort) comme étant un fait connu et acquis, sur lequel s'appuyer pour expliquer d'autres faits. Mais qu'est-ce que la réflexivité précisément : comment la définir, quel sens lui donner, et surtout quelle sorte de phénomènes nous fait-elle voir? Comment en avoir la conception plus claire et articulée possible, au-delà du foisonnement des termes et des approches? Aussi voudrais-je montrer qu'une vue générale sur la réflexivité conduit à la mise en question (et dans certains cas, au rejet) de nombreux termes et approches (le cas plus important concernant une bonne partie des approches qu'on appellera ici " méta " : celles qui raisonnement en termes de métalangage, métadiscours, métavisuel, métacinéma, etc.).

Mon but final n'est guère de trouver une clé de lecture ultime des sciences humaines, ni d'adopter une démarche qui, pour être trop généralisante et ambitieuse, écraserait la diversité des phénomènes à laquelle le savant ne devrait jamais être insensible. Bien au contraire, je voudrais montrer que c'est pour servir la nuance qu'une telle étude devient utile. Ainsi, interroger la réflexivité en général, comme nous le ferons dans la première partie de cette étude, nous servira à ne pas croire ou avoir recours à une conception des signes comme totalement référentiels ou réflexifs, entièrement transparents ou opaques (alors même qu'un grand nombre d'approches en sciences humaines ne fait que reproduire, sous-entendre ou présupposer une telle vue, délibérément ou non). Proposer des critères de distinctions entre les phénomènes réflexifs, comme nous le ferons dans la seconde partie, nous permettra d'analyser plus finement et clairement la conduite des acteurs sociaux, le parler ordinaire, le monde de l'art, bref tout ce qui, pour ainsi dire, est fait de sens.

\section{Premier temps : la généralité de la réflexivité}

L'omniprésence de la question de la réflexivité dans les sciences humaines suggère d'emblée qu' il y a réflexivité là où il y a du sens. Je voudrais reconvertir ici ce constat en parti pris théorique : la réflexivité est une manière de définir et d'analyser ce qu'on appelle le «sens », que ce soit le sens d'un comportement ou d'une œuvre.

Ce que je voudrais illustrer dans un premier temps, c'est qu'à chaque fois qu'on essaie d'approcher les faits de sens d'une manière un tant soit peu articulée - que ce soit au sein de la sociologie ou de la linguistique, de la philosophie ou de la sémiotique - on se sert d'une dialectique, on fait valoir une bipolarité, avec, d'une part, quelque chose qui est de l'ordre du direct, du visible, de l'immédiat et, de l'autre, quelque chose d'indirect, d'opaque, de médiat; et que ce second ordre de phénomènes se réfère au premier, en est le reflet, le repli, l’envers en quelque 
sorte. Une première manière de nommer les termes d'une telle dialectique ouverte peut être: d'une part, représentation et, de l'autre, présentation de la représentation. D'une part, on pose ce qu'on dit, on fait, on perçoit, l'objectalité en quelque sorte (ce que je nomme, très génériquement, représentation) et, de l’autre, la manière, l'orientation, l'intention, l'attention envers cette représentation (la présentation de la représentation). Nous verrons qu'il existe, en sciences humaines, un grand nombre de couples conceptuels qui redisent et reformulent toujours cette même dialectique générale. Aussi les faits que je voudrais monter en épingle sont-ils au nombre de deux. Premièrement, on ne s'est pas vraiment rendu compte de la prolifération terminologique et conceptuelle autour de cette même question, qui est la question même du « sens ». Deuxièmement, ce dont il s'agit à chaque fois, c'est une dynamique "réflexive», le second terme de chacun de ces couples étant le mode d'être $d u$ premier terme, son envers, quelque chose qui est autre bien que constitué de lui. C'est précisément ce qui se passe lorsqu'on prend, explicitement ou pas, un comportement en tant que blague, une discussion en tant que dispute, un geste en tant que provocation, une scène en tant que parodie, un récit en tant que fiction, une ouvre en tant qu'art; il est toujours question de comment prendre ce qui est figuré, représenté, référencié : dans quel sens, de quel côté. Or ce « côté » appartient bien au fait sémiotique en question, mais en même temps lui est irréductible, il est autre. L'énoncé « je suis désolé » peut viser à, peut avoir comme sens de s'excuser; or l'excuse, c'est le fait même de représenter soi-même comme étant désolé (d'où la réflexivité), bien qu'elle ne soit pas réductible à des mots de regret (elle est autre chose qu'un simple énoncé). De même, les performances d'artistes peuvent valoir comme étant de l'art; or, d'un certain point de vue, l'art est ce que les artistes performent, mais pas entièrement : seulement d'un certain point de vue (nous pouvons dire que l'œuvre d'art représente aussi quelque chose, qu'est présenté comme étant de l'art).

Ainsi la question de la réflexivité est-elle la question générale $d u$ sens de tout objet phénoménal (énoncé verbal, comportement social, œuvre d’art...), bien que chaque approche scientifique, chaque discipline et sous-discipline n'y voient à chaque fois, et dans le meilleur des cas, que des questions particulières (alors que dans le pire des cas, elles semblent aveugles aux questions réflexives mêmes, comme nous le verrons plus loin). En effet, force est de constater que rarissimes sont les études générales de la réflexivité; en sciences du langage, on compte notamment l'ouvrage du jeune Récanati, La transparence et l'énonciation ${ }^{7}$; en sciences sociales, le chef-d'œuvre de Goffman, Les cadres de l'expérience (le titre original est Frame Analysis) ${ }^{8}$. Le premier est une étude axée sur la tension entre transparence et opacité; le second, sur la tension entre expérience et encadrement (framing). La réflexivité est ici, encore une fois, dans le second pôle de ces tensions :

\footnotetext{
7. Récanati (1979).

8. Goffman (1974).
} 
dans l'opacification d'une référence verbale qui semblerait transparente; dans l'encadrement d'une expérience sociale prétendument immédiate. Nous allons partir de ces deux conceptions pour illustrer, d'abord, la réflexivité générale des faits de langage et, ensuite et plus brièvement, la réflexivité des faits sociaux. Pour finir cette première partie générale, on abordera de manière critique les approches «méta » et les approches « figurales».

\subsection{La généralité de la réflexivité à partir des faits de langage}

Nous commençons par les sciences du langage et avec l'ouvrage de synthèse de Récanati. Ce dernier, se proposant une mise au point de la tradition philosophique analytique au sein de la théorie de l'énonciation, démontre que, pour expliquer un grand nombre de questions, le langage ne peut être conçu que comme étant à la fois transparent et opaque. D'une part, le langage produit toujours une représentation : il est donc transparent, ou en d'autres termes référentiel. De l'autre, le sens de telles représentations ne se loge jamais tout à fait dans les représentations mêmes : il est plutôt dans l'activité de présenter ces représentations, de montrer ce qui est dit; en d'autres termes, le langage est aussi une présentation : il s'appuie sur soi-même pour fonctionner, il est donc opaque, ou en d'autres termes encore sui-référentiel.

Il y a au moins deux lignées d'études linguistiques qui confluent dans une telle conception. La première est celle des déictiques ou embrayeurs, la seconde est celle (en petite partie liée à la première) des actes de langage. Soient les embrayeurs, d'abord. On sait que le sens de « je » n'est pas tout à fait ce que « je » signifie en soi, ce qu'il représente, à savoir une " première personne singulière qui parle »; « je » n'a de sens que dans l'acte de quelqu'un qui s'empare de lui, qui prend la parole, dans ce qu'on peut appeler une sui-référenciation. La même chose se passe, bien sûr, pour tous les pronoms personnels de première et deuxième personne, ainsi que pour les « ceci », «i ici », " maintenant », pour les temps verbaux présent et passé, bref pour toute une famille de phénomènes où, clairement, la langue exhibe sa nature instrumentale et sui-référentielle. Instrumentale, parce que, selon les mots célèbres de Benveniste, «le langage est ainsi organisé qu'il permet à chaque locuteur de s'approprier la langue entière en se désignant comme je " ${ }^{9}$. Sui-référentielle parce que, de la sorte, elle "se réfère [...] à une réalité qu' [elle] constitue [elle-même] ${ }^{10}$. En somme, « je » et tous les autres embrayeurs sont bien transparents, en ce sens qu'ils produisent un référent (comme la première personne singulière signifiée par «je»); mais ils sont aussi opaques, dans la mesure où ils ne font sens qu'en se référant aussi à eux-mêmes en tant qu'outils pour produire un référent (comme le référent de la première personne qui parle en disant précisément « je »). Autrement dit, « je » et les autres embrayeurs sont réflexifs ${ }^{11}$.

9. Benveniste (1958, p. 262, souligné dans le texte).

10. Benveniste (1962, p. 274), sur l'énoncé performatif.

11. Pour une vaste étude de ces phénomènes, et la difficulté traditionnelle de les définir : Kleiber (1986). 
La linguistique dite de l'énonciation s'est développée dans une étude élargie des embrayages dans la langue. D’une part, elle a montré la diffusion de la subjectivation, à savoir de la possibilité et nécessité, pour le locuteur, de s'emparer de la langue et d'être représenté, figuré, par elle ${ }^{12}$. D'autre part, elle a porté l'attention sur la quantité innombrable d'expressions propres à la prise de parole qui, sans être de véritables embrayeurs, sont toujours des présentations de la langue en tant que telle, qui s'opacifie et devient clairement sui-référentielle; par exemple, on a étudié la famille ouverte des " comment dire? ", «à proprement parler », " voyezvous ", " ce qu'on appelle... », « je ne te dis pas », etc., qu'on a définis comme des boucles réflexives ${ }^{13}$. En général, cette tradition de la réflexivité dite marquée est aujourd'hui bien établie en sciences du langage, mais nous devons souligner que sa force constitue aussi sa limite : elle consiste en une recherche ponctuelle des faits réflexifs, après avoir montré que la réflexivité est une dimension diffuse de la langue. Aussi n'étudie-t-on que les marques de la réflexivité, c'est-à-dire les phénomènes linguistiques où la réflexivité est explicite, alors qu'on s'est rendu compte ou qu'on veut illustrer que la réflexivité est constitutive de tout fait de langage, peu importe sa formulation. (C'est là la chose essentielle que je voudrais soutenir dans cet article : bien qu'en théorie on hisse la réflexivité au rang de composant de tout fait de sens, en pratique on finit par ne s'attacher qu'à un aspect de la réflexivité même présent dans certains phénomènes, selon une démarche qui contredit ainsi son propre principe fondateur. Certes, la science se doit de faire preuve de précision et d'autolimitations, mais une vue trop focalisée et cadrée, partielle et parcellisée, ne devient-elle pas myope?)

À cet égard, comme le montre bien Récanati, la rencontre de la linguistique de l'énonciation avec les études sur les actes de langage est importante. L'entreprise qui fonde l'étude des actes de langage peut se résumer dans les termes d'une critique et dépassement de la tradition millénaire de l'" illusion descriptive » du langage ${ }^{14}$. Cela se fait, chez Austin, en deux temps. D'abord, on montre qu'une grande partie des actes de parole seraient incompréhensibles en tant que simples descriptions, constats; il s'agit alors de faire place à une famille d'actes de paroles nouvelle, inexplorée jusque-là : à côté des " constatifs », il faut s'attacher aux "performatifs ». Ensuite, on se rend compte de l'impossibilité de répartir les énoncés entre les constatifs et les performatifs, car en réalité il ne s'agit pas là d'une dichotomie de la langue, mais d'une tension interne à toute occurrence langagière. Toute parole

12. Cf. Kerbrat-Orecchioni (1980).

13. Authier-Revuz (1995). On peut aussi signaler que, dans une autre tradition, bien avant la linguistique de l'énonciation francophone, on s'est attaché à ce qu'on a appelé les glossing practices : l'ensemble des tactiques langagières qui, très régulièrement, permettent aux locuteurs de toute conversation de rattraper et en même temps exploiter le vague du langage, de dire ce qu'ils voulaient dire prétendument : cf. Garfinkel et Sacks (1970).

14. Austin (1962, p. 39 tr. fr.). Cf. aussi Austin (ibid, XI conférence), ainsi que Ducrot (1986, p. 176) et surtout Récanati (1979, chap. 6 « Réflexivité et niveaux de langage »). 
possède, d'une part, une dimension constative, qui consiste à simplement dire, se référer, signifier, et qu'Austin appelle alors dimension "locutoire »; et, d'autre part, une dimension performative, qui concerne l'efficacité du dire même, sa force, sa valeur, qui opacifie la signification et rend la parole un moyen d'action, et qu'Austin appelle dimension "illocutoire ". "Ce sont des phrases comme cellesci : 'Je m'excuse' et 'Le chat est sur le paillasson' [...] - cas extrêmes et marginaux - qui ont fait surgir l'idée de deux énonciations distinctes. Mais [...] tout acte de discours authentique comprend les deux éléments à la fois » ${ }^{15}$, c'est-à-dire la performativité évidente d'un «je m'excuse » et la constativité supposée d'un "le chat est sur le paillasson».

Ainsi, si je dis «j'ai oublié ton nom », «je cherche ton nom », voire «j'ai une mauvaise mémoire avec les noms ", " je commets toujours des gaffes avec des gens qui viennent de me dire leur nom ", à la fois je donne une certaine représentation de moi (selon la dimension locutoire) et je me sers de celle-ci pour agir, par exemple pour te demander de me dire encore une fois ton nom (selon la dimension illocutoire). Les deux éléments, aspects, dimensions coexistent; et le deuxième est précisément ce qu'on peut appeler le sens (réflexif) du premier.

Certes, il est des cas de réflexivité explicite : si je dis « je te demande à nouveau ton nom ", "quel est ton nom déjà? ». Mais ces cas, Austin l'indique clairement, sont trompeurs car " extrêmes et marginaux ». Les cas ordinaires consistent, par exemple, à ne pas formuler une question, ne pas l'exprimer en langue, grammaticalement, par des marques, mais plutôt à exprimer, dire, représenter quelque chose dont le sens est d'être une question. Un autre exemple : imaginons un conférencier qui s'adresse au président de séance avec ces mots-ci : «j'aimerais savoir l'heure ». Grammaticalement, aucune question n'est prononcée : on ne représente qu'un désir. Mais, sémiotiquement, on présente bien une question. La réflexivité de la langue serait donc ici le fait qu'on produit une représentation transparente, descriptive (le désir de savoir l'heure), pour s'en emparer de manière performative, pour accomplir un certain geste (l'interpellation opaque pour savoir l'heure). Il est clair à quiconque que, du président de séance qui ne réagirait pas, on pourrait dire qu'il n'a pas saisi le sens de l'énoncé ${ }^{16}$.

15. Austin (1962, p. 149 tr. fr.).

16. Les exemples que nous avons évoqués, tout comme d'autres qui suivront, sont typiques du domaine dit, en pragmatique, de l'implicite et du calcul interprétatif, où l'on distingue plus finement entre présupposés, sous-entendus, implications, insinuations, allusions, etc. (cf. Kerbrat-Orecchioni, 1986). Mon propos ici n'est pas de discuter ces questions directement, mais simplement de les convoquer, voire de les rappeler, pour pourvoir disposer d'un cadre vaste pour la problématique de la réflexivité, pour éclairer par la suite des phénomènes qui normalement sont associés à d'autres domaines (ainsi montrerai-je que l'art tire un grand profit des questions pragmatiques du sous-entendu, du malentendu, etc.). 
Le sens de ce qui se dit, c'est «comment il faut prendre " ce qui se $\operatorname{dit}^{17}$. Comprendre un énoncé, c'est toujours comprendre non seulement ce qui est dit, mais le geste que le dire constitue - que celui-ci laisse ou non des traces dans le dit. Ducrot est très explicite sur ceci : «l'étude des dialogues effectifs montre que l'enchaînement des répliques se fonde moins sur 'ce qu'a dit' le locuteur que sur les intentions qui, selon le destinataire, l'auraient amené à dire ce qu'il a dit. On répond à 'Il paraît que ce film est intéressant' par 'J'y suis déjà allé' [... Force est donc] d'admettre que le sens ne se déduit pas directement de la signification [grammaticale] $»^{18}$.

Ainsi la linguistique pragmatique mobilise-t-elle un (ultérieur) couple conceptuel : signification vs sens. La "signification » est la représentation produite par la langue (on peut l'assimiler à la dimension locutoire d'Austin); le « sens » est la présentation de cette représentation dans et pour une situation (on peut l'assimiler à la dimension illocutoire). Ainsi, pour reprendre l'exemple de Ducrot, « il paraît que le film est intéressant » signifie un certain jugement positif accordé au film; il y a bien donc la représentation d'un état de choses; mais son sens est le geste de se servir de cette représentation pour pousser l'interlocuteur à aller voir le film. Ainsi le sens est-il fatalement pratique, et donc réflexif : il se sert de ce qu'il est en train de produire, et qui est sa seule manifestation ${ }^{19}$.

Certes, il peut y avoir une réflexivité explicite, le sens peut être dit, le travail de l'énonciation être manifesté par des marques: «par ces mots, je t'invite à voir le film ", " ceci est une invitation ", "si je te dis que le film est intéressant, cela veut dire $q u^{\prime}$ il faudrait que tu ailles le voir ». Mais cela ne change en rien le fonctionnement réflexif de tout énoncé. Au contraire, cela ne fait que poser une question de plus : celle de la gamme des possibilités d'explicitation de la réflexivité et de sa traçabilité,

17. Ibid. (p. 93, 112, passim). Encore une fois, on trouve dans l'ethnométhodologie des propos tout à fait similaires, que la linguistique de l'énonciation semble avoir longuement ignorés : « reconnaître le sens de ce qu'une personne a dit consiste alors uniquement et entièrement à reconnaitre la méthode de son énonciation, donc à voir comment elle a parlé » (Garfinkel, 1967, p. 88 , souligné dans le texte).

18. Ducrot (1979, p. 97).

19. Cf. Ducrot : il faut se décider à « considérer le sens comme une description de l'énonciation. Ce que communique le sujet parlant au moyen de son énoncé, c'est une qualification de l'énonciation de cet énoncé. Idée paradoxale en apparence, puisqu'elle suppose que toute énonciation fait, par l'intermédiaire de ce qu'elle véhicule, référence à elle-même» (1984, p. 182). Pour le couple conceptuel signification/sens : cf. Ducrot (1984, pp. 180-185 et passim). Ce couple est aussi maintenu par d'autres approches linguistiques, telle que la linguistique interprétative de Rastier, pour qui le sens actualise non seulement les "sèmes inhérents ", c'est-à-dire les unités porteuses de "signification", mais aussi les "sèmes afférents ", c'est-à-dire les unités sémantiques qui émergent dans un cotexte, une situation, une pratique : cf. p. ex. Rastier (2001, p. 302). Il s'ensuit que Rastier donne une importance dominante au sens, considérant la signification comme un artefact positiviste (p. ex. Ibid., p. 11) : il s'en prend explicitement, et justement, à l'idée d'une existence de la signification en soi. Mais comme nous le verrons, il est important ne pas céder pour autant à la tentation de simplement renverser l'approche traditionnelle qui fétichise la signification : le sens n'a pas d'existence en soi non plus... 
dont nous nous occuperons dans la deuxième partie de cette étude. Ici, il importe d'admettre que tout fait de langage, tout ce qui fait sens est, en tant que tel, réflexif et transitif à la fois. Pour qu'un énoncé fonctionne en tant qu'invitation, il peut ou non énoncer "ceci est une invitation »; de même, pour qu'une fiction fasse sens comme étant une fiction, elle peut ou non signifier "c'est une fiction ». Ces remarques, pour banales qu'elles puissent sembler, s'avèrent extrêmement précieuses lorsqu'on se rend compte de combien il est courant de ne penser qu'il n'y a réflexivité que là où elle est marquée, énoncée, voire signalée ${ }^{20}$.

\subsection{La généralité de la réflexivité dans les images, les comportements, etc.}

Il convient d'admettre une tension entre la dimension représentationnelle et la dimension présentationnelle du langage - dans tout fait de langage. C'est cela même qui permet de définir la généralité des faits de langage, bien au-delà des phénomènes linguistiques. Aussi peut-on retrouver dans les images la même dynamique qu'on vient d'illustrer dans les paroles. Soit, d'une part, un dessin en perspective classique et, de l'autre, un dessin d'Escher. On peut dire que le premier est certainement plus transparent : il semble se limiter à représenter quelque chose; le deuxième est plus opaque : il affiche le fait d'être une construction graphique; bref, il est plus explicitement réflexif. Il n'en demeure pas moins que le premier, le dessin en perspective classique, fait toujours sens comme étant une construction graphique, et son spectateur le prendra toujours comme une image dessinée : personne ne s'est jamais cogné contre la plus réaliste des images. En effet, ce que de nombreuses approches oublient, c'est que c'est précisément en tant qu'images que de telles représentations sont réalistes. Et l'argument complémentaire vaut pour le dessin d'Escher : ce dessin ne se limite nullement à indiquer le simple fait d'être un dessin, car tout spectateur voit bien en lui des représentations d'escaliers, d'arcades, de bâtiments; et c'est précisément grâce à ces représentations, dans ces représentations, qu'il voit le paradoxe : des escaliers qui commencent là où ils finissent, des personnes qui le montent la tête renversée, etc. Toute image, en tant que fait sémiotique, est bien référentielle et sui-référentielle à la fois, transparente et opaque, signifiante et réflexive, bien qu'à doses différentes. La représentation, la transparence, et la présentation, l'opacité, sont toujours les deux faces du même

20. Ainsi p. ex. Vouilloux (2004, chap. IV « Dans l'œuvre : limites de la réflexivité ») peut soutenir qu' « il existe des œuvres réflexives et des œuvres non réflexives » (p. 133), là même où il épouse entièrement l'approche sémio-pragmatique à la Austin-Récanati, enrichie en plus de la logique de Port-Royal, selon laquelle penser, c'est en quelque sorte penser que l'on pense - tout comme pour les pragmaticiens dire, c'est en quelque sorte dire que l'on dit. Or, si une image ou une œuvre n'étaient pas réflexives du tout, elles seraient entièrement cette chose qu'elles représentent : elles n'auraient pas de sens précisément en tant qu'image ou œuvre. Comme l'a bien montré Marin, dans ses nombreuses études sur la peinture classique (que Vouilloux fait siennes), « représenter signifie se présenter représentant quelque chose » (1988, p. 255). 
fait de sens; voir l'une avant l'autre n'est qu'une question d'orientation du fait même - ou de la théorie ${ }^{21}$.

La théorie qui a sans doute le plus embrassé et exploré la complexité des faits de sens en tant que tels, à savoir en tant que réflexifs, est sans doute la frame analysis de Goffman ${ }^{22}$. Elle nous livre une quantité on ne peut plus riche et détaillée de cas, montrant, bien au-delà des simples énoncés verbaux, ce que les linguistes soutiennent pour la langue : si les comportements ou les situations, tout comme les énoncés, semblent posséder un aspect littéral, pré-interprétatif, il faut en réalité considérer celui-ci comme l'objet d'une activité d' « encadrement», dont on aurait tort d'ignorer la complexité. Nous sommes constamment en train de manipuler et tordre la prétendue littéralité de nos actions. Nous ne faisons, par nos actions, que plaisanter, leurrer, expérimenter, provoquer, imiter, parodier, répéter quelque chose avant de la faire pour de bon... Tous ces sens ne sont pas dans la prétendue littéralité du comportement : ils sont leur épaisseur nécessaire; ils ne sont pas de simples assaisonnements qui s'y ajouteraient : ils sont ce en quoi le comportement, en tant que tel, consiste. Le comportement est ainsi sui-référentiel (et c'est peut-être là le seul trait qui puisse permettre de le distinguer d'un mouvement quelconque du corps qui serait littéralement identique).

Ici, un malentendu doit être levé, qui vient peut-être de la tradition philosophique et psychologique qui a, la première, problématisé la réflexivité : qu'un comportement soit merveilleusement réflexif ne signifie pas qu'il est forcément conscient. Songeons à cet exemple ordinaire : nous allons au cinéma voir le film d'épouvante Shining; nous applaudissons Jack Nicholson dans le rôle de Jack Torrance, le fou épouvantable du film. Or, nous n'applaudissons pas le moins du monde les atrocités que Torrance commet (dans la représentation); nous applaudissons le fait que Torrance ait été performé si intensément (sa présentation). Bien que nous ne soyons face qu'à une seule et unique série d'actions, celles-ci font sens pour nous parce que nous les encadrons en tant que spectacle, et nous prenons donc Torrance-Nicholson comme une figure complexe qui a un aspect littéral, transparent, dans sa représentation de gestes abominables, et un aspect sui-référentiel, opaque, dans son admirable présentation cinématographique. La tension, l'entremêlement ou l'influence réciproque de l'un de ces aspects sur l'autre, est quelque chose d'important et difficile à démêler (car l'identité de l'acteur n'est pas imperméable à celles des personnages qu'il joue, pas plus que celles des personnages ne le sont par rapport à l'acteur - du point de vue esthétique, mais aussi éthique et même juridique...). Ce qui compte ici, c'est de convenir de

21. Encore une fois, il faut signaler ici que l'on doit à Marin (cf. 1989 et 1994) d'avoir expérimenté longuement cette approche pragmatiste, qui dérive notamment de la lecture de Récanati, dans le domaine des images picturales (cf. aussi Vouilloux, 2004, ch. III «À l'œuvre : l'usage de la représentation »). Pour une étude poussée de la réflexivité picturale mais précédente au tournant pragmatiste : cf. Marin (1977).

22. Cf. notamment Goffman (1974). 
la généralité de la réflexivité, du fait qu'elle dépasse de loin la présence à soi du spectateur, ou de n'importe quel type d'acteur social. C'est l'une des grandes leçons de Goffman : l'acteur social n'a pas à réfléchir volontairement à tout instant pour pratiquer ses jeux complexes de cadrage, pour que l'expérience fonctionne d'une certaine manière, en tant qu'expérience d'un certain type, selon un certain sens, bref de façon réflexive. (Par ailleurs, si tant est qu'on cherche à faire une place à la conscience de l'acteur social, ne serait-il pas plus opportun de le faire non pas dans le jeu réflexif du cadrage de l'expérience, mais là où celui-ci échoue ou achoppe, là où il $\mathrm{y}$ a erreur, problème, impasse?)

\subsection{La réflexivité manquée, notamment par l'approche "méta" et l'approche "figurale"}

Un autre malentendu doit être levé à propos de la réflexivité, et qui ne doit nullement être encouragé par les couples conceptuels qu'on est en train de passer en revue. Ce serait de penser que les énoncés verbaux, les comportements, les images, sont de deux types : référentiels ou sui-référentiels, transparents ou opaques, représentations ou présentations, expériences ou cadrages. Nous l'avons déjà pointé à maintes reprises, mais il s'agit là du propos central et délicat de cette étude. Premièrement, il (me) semble que jusqu'à présent on n'a pas vu à quel point les théories du ont en commun de se bâtir sur des couples conceptuels qui ne font que reformuler à chaque fois le même problème - mais en l'abordant à travers un nouvel angle. Deuxièmement, de tels couples sont conçus de deux manières opposées : soit comme des paires de cases, où répartir les phénomènes étudiés; soit comme des dialectiques qui animent tout phénomène de sens depuis l'intérieur. Autrement dit, la réflexivité est soit, dans le premier cas (majoritaire), résolue et dissolue, soit, dans le second (qu'on défend ici), valorisée et expliquée.

Ainsi, le couple représentation/présentation est-il abordé aussi, en esthétique analytique, dans les termes du voir/voir-comme, du dire (décrire, dépeindre)/ montrer, du dénoter/exemplifier (exprimer, évoquer ${ }^{23}$. Ainsi le couple signification/sens dérive-t-il, d'une certaine manière, de la conception qui oppose le répertoire (les erga) à l'activité (l'energeia) ${ }^{24}$; tout comme les couples énoncél énonciation, dictum/modus, représentation/opération de représenter ${ }^{25}$.

23. Pour ne donner qu'une seule référence pour ces couples, précieuse aussi pour la discussion qui suivra : cf. Goodman (1968, chapp. I « Refaire la réalité » et II « La sonorité des images »). Une excellente présentation et discussion critique des couples de Goodman (avec l'introduction de l'« évocation » comme autre mode de l'exemplification), ainsi que d'autres que nous citons, comme signification/sens ou dénotation/connotation, se trouve dans Genette (1991, chap. «Style et signification »).

24. On sait que l'opposition remonte à Humboldt (1836) et a eu une influence essentielle sur la théorie linguistique moderne, du côté du saussurisme comme de celui du chomskysme.

25. Ces oppositions remontent à Bally (1932, chap. premier « La phrase »). La théorie de la modalisation de Bally est tout à fait compatible avec celle de la force illocutoire des actes de langage (cf. Ducrot, 1986). 
Or, il est tout à fait appauvrissant, aussi bien dans la théorie que dans la pratique d'analyse de corpus, de maintenir qu'un fait sémiotique se situe soit au niveau de l'énoncé, soit au niveau de l'énonciation; un fait de langue, soit au niveau de la signification, soit au niveau du sens; ou encore, soit au niveau du sens dit littéral, soit au niveau du sens dit intentionnel ou dérivé; qu'il constitue soit un dictum, une dénotation, soit un modus, une expression; que finalement la langue entière se partage entre répertoire d'une part et activité de l'autre. Tous ces couples ne font que pointer la complexité constitutive de tout fait sémiotique, leur réflexivité. Mais la réflexivité est bien le pli qui n'existerait pas si les deux couches qui le constituent étaient séparées; si elles n'étaient pas le même pan qui se laisse prendre par deux côtés différents, qui se laisse voir en deux faces distinctes. Ouvrir le pan plié et n'y voir qu'une seule dimension ou, au contraire, le prendre du haut et n'y voir que deux niveaux superposés, c'est précisément perdre le pli ${ }^{26}$.

Prenons pour exemple représentatifle cas de la sémiotique structurale : puisque celle-ci sépare soigneusement le niveau de l'énoncé du niveau de l'énonciation; le niveau sémiotique de la dénotation du niveau sémiotique de la connotation, qui est successif au premier et l'enveloppe en bloc; puisqu'en somme elle opère par nivellements, elle manque les phénomènes réflexifs, et n'en fait même pas état ${ }^{27}$.

26. On sait que le «pli » a été hissé au rang de véritable concept philosophique par Deleuze, mais ici je me limiterai à la référence de Merleau-Ponty (1945, pp. 247-249, 476-479, 474-490 et passim), centrale pour penser la question de la réflexivité d'une manière générale et radicale. Très schématiquement résumée, cette philosophie essaie de penser autrement, de dépasser ou de se passer des grandes oppositions que sont subjectivité/objectivité, intériorité/extériorité, textualité/contextualité. En sciences du langage, on peut trouver une sensibilité de cette sorte lorsque Maingueneau propose une étude où «le discours ne fait qu'un avec la manière dont il gère sa propre émergence, l'événement de la parole qu'il institue; il représente un monde dont son énonciation est partie prenante. $\mathrm{Si}$, au lieu de considérer la seule cohérence/cohésion des œuvres, on saisit leur émergence comme événement énonciatif qui représente un monde tout en établissant les conditions de son dire, on met en cause la répartition traditionnelle des tâches entre une approche qui prendrait en charge l'extérieur' du texte et une approche du texte comme système conceptuel» $(1995$, p. 40); c'est dire que, par exemple, « il faut accéder à la situation d'énonciation que toute œuvre constitue par son déploiement même, la situation dont elle prétend qu'elle la rend légitime et qu'elle légitime en retour [...] en traçant une boucle paradoxale [...] » (p. 54). On trouve une sensibilité opposée, et à cet égard critiquable, dans l'étude des actes de langage qui tend à distinguer ontologiquement ce qui est dit dans la langue de ce qui est porté par l'intentionnalité du sujet parlant, ce qui est dans une convention partagée de ce qui est dans l'esprit de tout un chacun : c'est la manière dont Searle, d'après Grice, reformule la pragmatique d'Austin dans une direction mentaliste (cf. parmi d'autres critiques : KerbratOrecchioni, 1986, p. 313 ss.).

27. Je me réfère à la sémiotique qui va de la théorie classique de l'empilement des sémiotiques du Hjelmslev des Prolégomènes (1943) jusqu'aux travaux expérimentaux de Fontanille (2008) sur le nivellement hiérarchique des niveaux de pertinence des signes/textes/objets/pratiques/etc. Le centre de cette sémiotique est, bien sûr, l'œuvre de Greimas, où l'on peut relever l'intérêt somme toute très restreint pour la question l'énonciation, conçue presque seulement à travers deux articulations parallèles : le rapport compétence/performance et le nivellement énonciation "proprement dite »/énonciation énoncée (ou débrayée) (cf. notamment Greimas et Courtés, 
Aussi me semble-t-il utile de pointer ici une série de phénomènes réflexifs qui sont ignorés typiquement par la sémiotique structurale. Cela me permettra, d'une part, d'expliquer d'une manière plus fine les dynamiques représentation/présentation, référence/sui-référence, dimensions locutoire/illocutoire, signification/sens...; d'autre part, de parvenir à une critique des nombreuses approches qui, même lorsqu'elles acceptent éventuellement l'idée d'une réflexivité (ne fût-ce qu'une réflexivité ponctuelle, marquée), fonctionnent au fond soit par nivellements soit par mises à plat, et manquent ainsi le pli des faits de sens, leur nature réflexive.

Le premier type de phénomènes réflexifs invisibles à toute approche fondée sur la transparence du sens, sur le privilège de la signification, de la figuration, de la représentation, est ce qu'on peut appeler le sens obscur. Il ne s'agit pas, ici, de la pratique exégétique (d'où cette appellation provient); il s'agit plutôt des cas ô combien ordinaires - où l'on ne saisirait que la dimension référentielle d'un comportement, d'un spectacle artistique, d'un propos oral, sans que l'on comprenne où ces faits sémiotiques en situation "veulent en venir ", ce qu'ils "veulent dire par là». Il faut que nous disposions d'outils pour expliquer que, d'une part, on puisse saisir ce qu'on représente, par exemple l'espace-temps unitaire figuré par le long plan-séquence d'un film; et que, de l'autre, un problème réflexif s'ouvre, par exemple le sens de cette construction spatio-temporelle même, de ce planséquence en tant que tel, dans tel film, dans tel moment (il s'agit d'un problème abordé normalement, mais d'une manière pas assez fine, en termes de style ${ }^{28}$ ).

Un deuxième ordre de phénomènes que nous devons pouvoir analyser est ce qu'on pourrait appeler le malentendu. Comme dans le cas du sens obscur, dans le malentendu on saisit ce qui est énoncé, mais en plus le sens semble être immédiat; le geste de l'énoncé, transparent; or, cette transparence, cette immédiateté va basculer ensuite. D'un certain point de vue (du point de vue de l'observateur situé pour qui seulement le malentendu fait sens) ${ }^{29}$, le malentendu est le retardement de la multiplication des sens qui affectent un même fait, un même référent, une même signification. Imaginons que notre copain de sortie nous dise, au comptoir, quelque chose de bien transparent, descriptif : « je n'ai pas de sous »; et imaginons que cet énoncé, dont la signification ne nous échappe pas, nous le comprenions comme une demande à ce qu'on l'invite. Imaginons alors que cette parole s'avère être une moquerie qui nous est adressée, car nous sommes censés dire toujours à notre copain « je n'ai pas de sous». Ou qu'elle s'avère être simplement une moquerie

1979, «énonciation » et passim). Mais les oppositions tranchées compétence/performance et énonciation/énonciation énoncée semblent, depuis quelque temps, avoir atteint leurs limites heuristiques, en partie pour les raisons que je suis en train d'exposer ici.

28. Cf. toujours Genette (1991, chap. «Style et signification »).

29. Je dis bien qu'à l'observateur situé le sens semble immédiat, transparent; alors qu'à l'observateur distancié que nous sommes ici, bien sûr, le sens des ces cas d'interactions demeure le produit d'un calcul interprétatif, comme on l'appellerait en pragmatique linguistique, ou d'un "framing», comme le nomme Goffman en pragmatique sociologique. 
que notre copain adresse à soi-même, puisque, effectivement, il n'a jamais d'argent quand il sort, mais nous avons toujours eu la gentillesse de ne pas le dire. Ou imaginons encore que le frame, le sens, s'avère être tout autre : un argument pour sortir chercher de l'argent dans un distributeur. Du point de vue de l'acteur, le malentendu est un reframing a posteriori, un sens que, pour ainsi dire, sera vu s'avérer (d'où son grand effet réflexif).

Tout comme le sens obscur n'est nullement l'apanage de l'interprétation philologique d'un texte, le malentendu n'est nullement un accident de la partie plus négligeable de nos activités : la sémiotique des images et de l'art les cultivent et valorisent assidûment. Mais ces sémiotiques manquent quelque chose d'essentiel si elles ne font pas valoir non seulement le fait qu'on se trompe de sens à un tel moment de la lecture, mais aussi, plus finement, que la signification de ce moment même, elle, demeure stable à travers les sens qui l'affectent ensuite. Autrement dit : qu'il y a quelque chose qui reste transparent à travers la modulation de l'opacité sémiotique; qu'il y a bien une tension entre signification et sens, référence et suiréférence, locution et illocution internes à la parole. Le «je n'ai pas de sous » de notre copain au comptoir signifie bien la même chose dans toutes les situations que nous avons évoquées, il garde une même dimension locutoire et une même référence; c'est pourquoi nous avons besoin de dire que c'est le sens qui change, la dimension illocutoire, la sui-référence; c'est pourquoi nous avons besoin de deux dimensions, internes à la parole en question. Prenons quelque chose de bien connu au cinéma : la femme fatale du film noir. Si je vois dans un film qu'une femme est extrêmement fascinante, douce, et qu'elle se fait aimer par le protagoniste qui devient prêt à tout pour elle, ce n'est pas la découverte successive des doubles jeux de la même femme qui va altérer la figuration d'une femme qui, oui, s'est fait aimer par le protagoniste et, oui, était extrêmement douce avec lui. Ce qui change est autre chose : c'est ce qu'on appelle, d'ordinaire, le sens de tout cela, la manière dont nous devons «le prendre " (tout comme nous devons "prendre " le « je " du langage verbal, nous "en emparer»), bref la dimension réflexive. Expliquer la sémiotique de l'art, c'est rendre compte de tels phénomènes, jouissifs ou bouleversants : le fait qu'on glisse sur une dimension (réflexive), alors qu'il y en a une autre (figurative) qui nous semble bien solide, bien que, précisément, nous ne sachions pas comment «la prendre». (A contrario, supposer un sens transparent, donné de manière synchronique, voire achronique, c'est se vouer à manquer des phénomènes essentiels.)

On objectera ici que les exemples précédents laissent croire, tout de même, que notre dimension réflexive, sui-référentielle, vient après la dimension figurative, référentielle; et qu'alors elle reste, somme toute, une couche qui s'ajoute : la couche problématique et seconde, par rapport à la couche du départ, qui serait alors la couche donnée et première. Si tel était le cas, en effet, on n'aurait pas à rejeter avec tant d'empressement la théorie des niveaux sémiotiques et de l'empilement des sémiotiques dénotative et connotative (et de la métasémiotique) : à partir d'une 
signification-dénotation, on aurait ensuite des effets de sens-connotation (et la construction, par là, d'une métasémiotique). Or, je pense qu'il n'en est rien. Le troisième ordre de phénomènes réflexifs que je voudrais évoquer devrait l'illustrer d'une manière très simple; il s'agit de ce qu'on appelle deviner. Songeons au cas où nous connaîtrions très mal une langue, sa grammaire et son lexique, mais nous comprendrions bien l'interaction qui est en train de s'y jouer. Or, on peut rendre compte de cela en disant que le sens, notre réflexivité aiguë, nous permet de surmonter nos problèmes concernant la signification des énoncés. Encore une fois, ce type de phénomène concerne des expériences ordinaires, par exemple lorsque nous voyageons dans certains pays, ou que nous apprenons une langue d'une manière totalement inductive, tout comme des expériences esthétiques, par exemple lorsque nous sommes face à l'art abstrait, ou l'art expérimental, ou toute forme d'art, même classique, qui demande, comme on dit, une forte participation du lecteur ou du spectateur.

En conclusion, premièrement, nous avons intérêt à distinguer la dimension référentielle, ce que le langage figure, de la dimension sui-référentielle, ce que le langage produit grâce à ce qu'il figure. Deuxièmement, nous devons voir qu'aucune de ces dimensions n'existe sans l'autre, contrairement, par exemple, à la dénotation face à la connotation, ou au constatif face au performatif. Selon les situations empiriques, selon les perspectives épistémologiques, l'une de ces dimensions peut paraître comme dominante par rapport à l'autre, mais il s'agit bien de deux dimensions d'un même fait et pas de deux types distincts de faits sémiotiques, deux aspects d'un même objet de sens et pas deux niveaux (nous pouvons très bien saisir ce que la sémiotique appelle la connotation avant de construire éventuellement ce qu'elle appelle la dénotation : c'est pourquoi, entres autres, l'épistémologie des empilements est pauvre pour l'analyse).

En sciences humaines, il est tout un vocabulaire qui, précisément, empêche de penser un tel dynamisme, que ce soit par un parti pris et assumé (comme lorsque, effectivement, l'approche «méta » fut mise en place dans la logique pour trancher la complexité sémiotique) ou par une forme de paresse face à la tradition qui découle de ce parti même (comme lorsqu'on continue de parler en termes de « méta » ou de " niveaux » sans partager forcément le parti logiciste). Je terminerai cette section en discutant brièvement de ces approches qui manquent la réflexivité, notamment des deux qui semblent les plus répandues et représentatives.

La première approche, je l'ai déjà évoquée comme le «méta». Rien de plus banal : on approche tel commentaire comme du «métalinguistique», telle mise en abyme comme du "métadiscours", telle discursivité des images comme du «métavisuel ». Le propre de ce parti, je l'ai suggéré à travers le cas illustre de la sémiotique structurale, c'est de supposer une transparence totale des faits de langage : de concevoir ceux-ci comme autant de représentations éventuellement entassées, hiérarchisées. Le parti «méta » consiste, précisément, à introduire une référence de second niveau là où l'on rencontre la sui-référence, qui est ainsi tout 
simplement faite disparaître. Lorsqu'on ne peut pas se trouver à un seul niveau de référence, de représentation (p. ex. $A$ représente « $\mathrm{x}$ »), on ajoute un deuxième niveau, dont le but est de se référer au premier, c'est-à-dire de représenter la représentation (p. ex. méta- $A$ représente « $A$ qui représente $\mathrm{x}$ »). Un tel parti, maintenu de manière cohérente, implique une démarche de ce genre : une scène de film qui cite ou parodie un autre film produirait du « métacinéma », c'est-à-dire il figurerait la figuration d'un autre film, il représenterait la représentation du monde du cinéma. Elle ne serait donc plus la figuration du film même, la représentation d'une histoire en cours par exemple. Et à partir de là le film entier serait donc à départager, niveler, en une figuration, une représentation, $A$ d'une part et une figuration, une représentation, méta- $A$ de l'autre. Mais la réflexivité est bien le double jeu de construire une figuration filmique et en même temps et toujours présenter celle-ci d'une manière cinématographique, artistique - ou aussi éthique, politique, etc. Tantôt ce sera l'une de ces dimensions à l'emporter éventuellement, tantôt l'autre; par exemple, tantôt, dans la lecture plus référentielle, représentationnelle, figurative, on peut arriver à ignorer la parodie ou la citation du film sans problème pour sa signification; tantôt, dans la lecture plus sui-référentielle, présentationnelle, réflexive, on peut s'attacher à la parodie ou la citation sans pouvoir décider de l'étendue exacte de la figuration, de la signification filmique concernée (comme lorsqu'on est face à quelqu'un qui, par ses dires, rigole sans qu'on ne sache où la rigolade même commence et finit, quelle étendue de signification est affectée par le sens " rigolade »). Nous avons à saisir la complexité sémiotique de ce double jeu, et non à la distribuer sur une partie de premier degré et sur une autre de second, sur une composante référentielle et une autre sui-référentielle, sur un niveau dit de l'énoncé et un autre dit de l'énonciation. Nous devons convenir qu'il est bien plus heuristique de voir qu'un seul et même plan se tord, se plie, consiste en deux dimensions. Un exemple on ne peut plus banal - et pourtant déjà bien complexe pour nos outils : la fille du parrain qui est figurée, représentée à la fin de la célèbre saga cinématographique de Francis F. Coppola, The Godfather. Le fait que celle-ci soit la fille même du réalisateur, Sophia Coppola, est-ce indiquer que celui-ci est un (le) parrain? Dire que la fille du parrain (dans le référent interne au film) est un individu et la fille Coppola (dans le référent méta du film) en est un autre, et mettre le premier à un niveau inférieur au second, comme si celui-ci était indifférent à celui-là - de même que le métalangage de l'algèbre est indifférent aux grandeurs qu'il peut subsumer - , ce n'est pas expliquer du tout le court-circuit sémiotique qui se joue ici; c'est a fortiori ne pas voir sa nature allusive, indirecte, ambiguëe c'est en somme s'empêcher de penser le problème même de la réflexivité ${ }^{31}$.

30. Cette question de l'indécidabilité de la dimension réflexive a été suggérée récemment avec finesse dans la théorie littéraire par Fraisse (2011).

31. Pour un exemple ultérieur : cf. n. 45. Pour une argumentation plus développée contre la logique classique, avec ses « niveaux de langage » et sa "phobie de la réflexivité » : cf. Récanati (1979, respectivement pp. 77-88 et pp. 25-27). Récanati insiste à montrer comment le propre de la 
Le parti représentationnaliste a tellement semblé usurper le domaine sémiotique qu'un autre parti s'est alors constitué en renversement radical, et même en opposition déclarée à toute sémiotique. Il s'agit d'un présentationnalisme radical, qui déjoue la prétendue transparence sémiotique en faveur d'une opacité définitive du sens. C'est un parti qui a pris un rôle de premier rang, notamment dans le pays du structuralisme, et notamment dans les études de l'art et des images en mouvement (notre discussion précédente sur la sémiotique structurale et l'art nous fait comprendre pourquoi). Ce parti s'attache à ce qu'on appelle le « figural » depuis l'ouvrage fondateur de Jean-François Lyotard : Discours, figure ${ }^{32}$. Comme le titre de l'ouvrage l'indique, il s'agirait d'opposer la « figure " au « discours ", sur l'idée que ce dernier est représentationnel, logique, systémique; la figure est alors énergique, pulsionnelle et résistante à toute grammaticalisation et systématisation. Et alors que le domaine de prédilection du discours serait la parole, lieutenant de la langue, le domaine de la figure serait notamment l'image, qui échapperait à toute langue. La figure ne peut pas être prise dans le système d'une langue parce qu'elle serait incarnée, matérielle. Ainsi, si la discursivité est référentielle et transparente, la figure serait opaque et événementielle. En somme la figure, notamment l'image, est, pour l'essentiel, l'expression d'un moment de forces, de désirs et de tensions.

L'approche figurale est loin d'être inintéressante pour la sémiotique et les phénomènes réflexifs, mais à la condition précise d'être vidée de son aspect quasi mystique, qui consiste à hypostasier l'« espace sauvage » de l'image, à attribuer une réalité autonome à ce que Freud appelle les « processus primaires », c'est-à-dire le monde des pulsions. Si l'approche figurale est heuristique, c'est seulement, encore une fois, à la condition de ne pas couper le sens en deux mondes, mais de l'innerver sur deux dimensions; de ne considérer donc la dimension figurale que comme une torsion de la dimension figurative; les forces, comme une résistance matérielle aux formes; la figure, comme une rhétorique et une pragmatique du discours - comme déjà Christian Metz avait pu l'argumenter dans la théorie du cinéma ${ }^{33}$

logique classique est le «représentationnalisme» : "pose[r] en principe que l'énonciation doit être mise entre parenthèses pour que l'énoncé puisse représenter un état de choses» (ibid. p. 121); suivre "cette illusion descriptive si commune en philosophie [qui] consiste à faire de la représentation la fonction essentielle du langage; en particulier [...] triturer les faits linguistiques de façon à en rendre possible l'interprétation en termes de 'représentation' ou de 'description' » (ibid. p. 96, en citant Austin; cf. en général chapp. 5 « Performativité et réflexivité » et 6 « Réflexivité et niveaux de langage »).

32. Lyotard (1971). Cet ouvrage a relancé et conceptualisé, dans l'esthétique, la question de Freud des « conditions de figurabilité » et des " procédés de la figuration », dans le « travail du rêve » et en général dans les processus primaires, pulsionnels (cf. notamment Freud, 1899, ch. IV, \$ IIIIV). L'impact de cet ouvrage a été remarquable dans les études de cinéma à vocation esthétique, lesquelles ont encore aujourd'hui une certaine résistance envers l'approche sémiotique. Pour un panorama actuel sur la filiation lyotardienne : cf. le recueil Aubral et Château (éds, 1999); pour une reconstruction des étapes majeures de cette tradition : Vancheri (2011).

33. Metz (1977, ch. IV « Métaphore/métonymie, ou le référent imaginaire »). 
et Gilles Deleuze le montrer dans son étude de la peinture ${ }^{34}$. Le figural, entendu non pas comme une réalité mais un aspect, un procès, une dimension, qui opacifie la référentialité des langages, valorise leur présence pratique, ouvre un écart par rapport à la signification grammaticale, est alors un cas particulier de réflexivité, que j'essayerai de préciser par la suite, dans la typologie des phénomènes réflexifs. En revanche, dans sa version ontologisante (la version plus lyotardienne), le figural est un simple renversement épistémique du "méta " : il hérite et entérine l'idée classique du langage comme représentation, et dès lors avance l'existence de quelque chose de sensé qui n'est plus du langage. L'image, pour les lyotardiens, n'est pas discursive ${ }^{35}$. Des représentationnalistes du "méta " au présentationnalistes du « figural », on passe de la transparence pure à l'opacité totale, de la référence absolue à la sui-référence aveugle. Pour les représentationnalistes du «méta », il n'y a que du discours sur, et donc il n'y a au fond aucune autonomie des faits de sens en tant que tels; pour les présentationnalistes du « figural », il n'y a que de la présence en soi, et donc aucune capacité des faits de sens de signifier quelque chose.

\section{Deuxième temps : les différences dans l'ensemble des phénomènes réflexifs}

Que la réflexivité soit générale, présente dans tout fait sémiotique, partie prenante $\mathrm{du}$ sens des discours ordinaires, des images, des comportements, cela ne veut pas dire que tout est toujours réflexif : on l'a vu, la réflexivité n'est qu'un aspect de ces faits mêmes. La généralité de la réflexivité signifie moins encore que tout est réflexif de la même manière : on établira maintenant qu'il faut opérer des distinguos au sein des phénomènes réflexifs. Notamment, je crois qu'il est essentiel d'en distinguer les différentes présences et orientations. Les modes de présence premièrement : la réflexivité peut être plus ou moins explicite, d'une part, et plus ou moins localisée, d'autre part. Les orientations différentes deuxièmement : la réflexivité peut être plus générique ou plus singulière. En dressant ainsi en tableau qui s'articule sur un axe des manifestations et un axe des orientations, je ne me pose, ici, que le but de fournir des critères simples et rationnels pour faire un peu d'ordre dans les études de la réflexivité, qui sont (à mon sens) trop spécialisées et réductionnistes.

34. Deleuze (1981). On peut citer aussi, dans cette même épistémologie du figural non ontologisé mais « dialectisé » : Didi-Huberman (1990; pour une compatibilité avec le structuralisme : cf. p. ex. p. 204); et aussi, référence importante dans les études cinématographiques : Aumont, dont l'étude figurale A quoi pensent les films? (1996) porte précisément sur la réflexivité des films, sur les « problèmes » que ces derniers essayent de " résoudre ».

35. Cette conception d'un sens non dicible, ou d'un dire sans dit, est en effet une nouvelle édition de l'esthétique romantique. D'ailleurs, Rancière (2004) a pu montrer aisément que l'esthétique de Lyotard est une réactualisation radicale de l'esthétique du sublime. 


\subsection{Premier axe, les manifestations : réflexivité diffuse/ponctuelle/globale}

La réflexivité est partout, mais selon des modes et à des degrés de présence bien différents. Il est clair qu'il y a des lieux et des moments où la réflexivité apparaît plus explicitement, peut-être plus intensément; en tout cas, de manière bien localisable. Nous en avons fait état lors de notre discussion linguistique : la réflexivité se manifeste avant tout dans des traces (si bien que c'est sur elles que les linguistes de l'énonciation concentrent la plupart de leurs efforts). Dans l'activité des échanges verbaux, on parle notamment d'embrayeurs; mais il est d'autres embrayeurs dans d'autres activités : par exemple, dans l'activité spectaculaire, le rôle d'embrayeur est joué par ce dispositif matériel qu'est la séparation scénique, ainsi que par cette pratique obligée qu'est l'applaudissement au performeur qui s'avance au bord de la séparation scénique. En effet, dans l'applaudissement, les participants des deux côtés de la séparation scénique, les performeurs et les publics, énoncent, de manière localisée, marquée, que ce qui a été représenté a fait sens comme une présentation spectaculaire : l'acteur de théâtre, par exemple, a fait sens en tant que personnage dramatique; son action, en tant que drame ${ }^{36}$.

Lorsque nous sommes face à des traces, qui explicitent la réflexivité d'une manière locale, on parlera de réflexivité ponctuelle. Or, l'étude de telles traces a montré qu'il ne s'agit là que d'autant de manifestations d'une réflexivité diffuse, qui est susceptible d'être localisée à tout moment. C'est dire qu'il faut prendre acte qu'il existe un continuum entre la réflexivité d'un fait sémiotique en tant que tel (ce qu'on appellera donc réflexivité diffuse) et la réflexivité qui trouve des figures et des procédés pour s'expliciter (les traces de la réflexivité diffuse, la réflexivité ponctuelle). C'est ce qu'a démontré, entre autres, Christian Metz, dans son étude essentielle de sémiotique du cinéma, L'énonciation impersonnelle ${ }^{37}$ : d'une part, un film peut faire un usage énonciatif, réflexif, de n'importe lequel de ses éléments (de la composition du cadre à l'éclairage, de l'emploi des sons au recours aux écritures sur les images, etc.); mais de l'autre, il existe aussi un ensemble de figures et de procédés culturellement stables pour cette entorse énonciative du film (par exemple, les adresses au spectateur de la part d'un personnage ou de la voix narrative, les écrans dans l'écran, etc.). En d'autres termes, d'une part, l'énonciation filmique est partout : tout élément peut être considéré comme énoncé et donc produit et trace, marque, d'une énonciation (n'importe quel cadre, en tant que produit d'un cadrage, ou n'importe quelle lumière, en tant que produit d'un éclairage, etc.); de l'autre, il existe bien un répertoire historiquement ouvert de figures et procédés de réflexivité reconnue, concentrée (seulement certains cadrages, ou certains éclairages : ceux qui feraient "faute voulue ", ou "clin d'œil au spectateur", ou «citation », ou «parodie », etc.); la réflexivité est alors discernable plus aisément puisqu'elle est

36. Sur l'importance réflexive du moment des applaudissements dans l'ensemble de la sémiologie spectaculaire, je me permets de renvoyer à une étude précédente : Tore (2011).

37. Metz (1991). 
attestée, localisée. Mais reconnaître que ce répertoire est historiquement ouvert, ou variable selon les situations et les pratiques particulières, c'est dire qu'il est illusoire de vouloir l'expliquer dans l'absolu, de le figer et l'élever au rang du seul ensemble des phénomènes réflexifs.

Expliquer la réflexivité, c'est bien distinguer une réflexivité diffuse et toujours localisable d'une réflexivité marquée et localisée ponctuellement. Mais c'est aussi faire place à un troisième type de réflexivité, plus complexe, encore moins évidente : une réflexivité qui est diffuse sans pouvoir devenir localisable, une réflexivité qui n'est pas susceptible d'emprunter des figures et de procédés qui l'explicitent, une réflexivité qui est simplement transversale et qu'on peut appeler globale. La réflexivité globale, en effet, ne fonctionne qu'en mobilisant la globalité du texte ou de l'événement sémiotique en question : elle ne se trouve que dans le sens qui résulte du rapport entre ses éléments, dans leurs tensions, leur dynamique, leur « texture ». Je suggérerai que, pour cette capacité de faire bloc, cette réflexivité est modélisante; et en outre, elle est caractéristique des sémiotiques de l'artistique et du spectaculaire ${ }^{38}$.

\subsection{La réflexivité globale, ou modélisante : réflexivité figurale/exemplaire}

La réflexivité globale est modélisante : on peut la définir, dans une première approche, comme ce que les textes, les œuvres, les événements sémiotiques montrent à travers ce qu'ils représentent dans leur globalité, dans leur ensemble. Il est deux sortes, très importantes, de réflexivité globale ou modélisante : la réflexivité figurale et la réflexivité exemplaire.

Commençons parla réflexivitéfigurale. Celle-ci, on le soulignait précédemment, demeure trop souvent objet de méprises et confusions, à cause desquelles on ne voit pas que le figural est, précisément, un phénomène réflexif. Le figural, en dehors de toute mystique de la révélation esthétique, peut être défini comme la logique une logique sui generis - de la représentation d'une œuvre. Elle n'est pas ce que l'œuvre dit, figure (par exemple, telle histoire d'amour dans un mélodrame), mais le modèle de ce qui est dit, le fonctionnement structurel de ce qui est figuré (par exemple une certaine violence, ou un certain schéma farcesque, ou une certaine dynamique d'indécidabilité, dans l'histoire d'amour mélodramatique). C'est un « raisonnement » interne à l'œuvre à travers la figuration de cette dernière. Dans la tradition occidentale, le type classique d'une telle sémiotique qui « raisonne par figures » sont les paraboles religieuses (et il est certain que cette sémiotique a largement influencé la sémiotique de l'art). Les paraboles possèdent bien un sens, une vérité sui generis, qui n'est pas dans le référent transparent que sont leurs histoires, mais dans ce qui à travers les histoires mêmes est montré de manière opaque. De

38. Pour une première approche de l'auto-modélisation des faits sémiotiques, et notamment des faits artistiques : Beyaert-Geslin et Fontanille (éds, 2003; notamment l'article de J. Fontanille, «Énonciation et modélisation », pp. 109-133). 
même, le sens figural d'une image n'est pas ce qui est, à proprement parler, localisable, dans l'image, dans sa figuration, mais il est la résultante des tensions et des rythmes, des pleins et des vides, qui traversent la figuration faite peinture, faite film, faite photo et, littéralement, en ressortent. Ainsi, dans une vaste étude figurale sur l'œuvre de David Lynch, Pierluigi Basso a pu illustrer comment le sens résultant de ces films est une expérience de mondes qui ont des manques et donc s'interpénètrent; c'est dire que l'œuvre de Lynch montre, figuralement, à travers ses différentes histoires dans ses différents films, l'interpénétration, des objets, des vies, des valeurs, des univers, leur conversion réciproque, leur réversion ou aversion; c'est là un véritable modèle sémiotique interne à l'œuvre de cet auteur ${ }^{39}$.

La réflexivité figurale est quelque chose d'assez vague, dira-t-on. Oui, en effet; mais ce n'est pas une raison pour ne pas essayer de la formaliser : nous avons à prendre bien au sérieux une véritable sémiotique du vague - comme l'a appelé et étudié en premier Peirce ${ }^{40}$. Il s'agit, toujours selon les termes de Peirce, de quelque chose qui fait sens en tant qu'éventail de possibilités, qu'ensuite différents parcours peuvent spécifier. C'est le fonctionnement de ce que Peirce appelle les icônes. Les icônes sont vagues puisqu'elles sont des qualités, et donc des possibilités; elles ne sont pas encore des faits, comme ce que Peirce appelle les indices, ni des lois, comme ce qu'il appelle les symboles; mais c'est précisément pour ce manque de détermination que les icônes sont précieuses et puissantes. Certes, elles «n'assertent rien " : c'est le symbolique qui asserte, et qui spécifie le vague de l'iconique; mais elles sont bien «le seul moyen pour communiquer directement une idée » : c'est l'iconique qui fournit au symbolique les prédicats qu'il va asserter ${ }^{41}$. Il s'ensuit que « une propriété grandement distinctive de l'icône est que, par son observation directe, peuvent être découvertes des vérités qui concernent son objet et qui sont autres par rapport à celles qui suffisent à déterminer sa construction $»^{42}$. Or c'est là ce qui se passe dans la réflexivité figurale. La réflexivité figurale n'asserte rien, elle consiste dans la carte des parcours où l'on pourra asserter, explorer et trouver, et même découvrir. On appelle « diagramme » une telle carte des possibilités, une telle formule des relations susceptibles de se réaliser d'une manière ou d'une autre ${ }^{43}$.

39. Cf. Basso Fossali (2006). Pour une analyse et une explication plus approfondie de la réflexivité figurale et sa capacité modélisante, à partir du corpus de Lynch : Tore (2008) - où cependant j'épousais encore la théorie d'une sémiotique connotative ou de deuxième niveau, qui aujourd'hui ne me semble plus assez fine.

40. Sur la sémiotique du vague : cf. Peirce (1905, p. 112 ss.), ainsi que la riche discussion dans Tiercelin (1993, chap. 5 « La sémiotique du vague »).

41. Peirce (1931-58, respectivement 2.291 et 2.278 ; éd. orig. : 1893 et 1895 ; nous traduisons).

42. Peirce (1931-58, 2.279; éd. orig. : 1895; nous traduisons).

43. Peirce (1931-58, 2.277; éd. orig. : 1903) distingue trois types d'icônes interprétées (ou hypoicônes) : les images, les diagrammes et les métaphores. Les diagrammes - ce dont nous traitons ici - sont définis comme des icônes de relations : ils montrent les rapports, ils modélisent le fonctionnement d'un ensemble. Sur la sémiotique des diagrammes, parmi les plus passionnantes chez Peirce : cf. Id. (1906). 
L'illustre étude figurale de Deleuze sur l'œuvre de Francis Bacon met en avant, précisément, de telles questions diagrammatiques ${ }^{44}$.

Cela étant pour la figuralité ou diagrammaticalisation, il existe un autre soustype de réflexivité globale et non localisable, sans traces ou procédés : l'exemplification, à savoir le procès par lequel une chose fonctionne comme un exemple de ce qu'elle possède. Elle aussi est caractéristique des œuvres d'art et des spectacles. Mais autant la réflexivité figurale est souvent objet de méprises, autant la réflexivité exemplaire est constamment méconnue dans l'esthétique - comme le soulignait déjà Nelson Goodman, qui a le plus travaillé sur le concept d'exemplification ${ }^{45}$. Il semble qu'on ne s'est pas assez rendu compte de combien un tel procès constitue non seulement une forme de réflexivité de premier ordre, mais aussi, sans doute, sa forme plus répandue, celle qui est susceptible d'embrasser la quantité la plus variée de phénomènes. C'est pourquoi il me paraît opportun de la présenter au sein de cette partie typologique, plutôt qu'à côté des études théoriques de la première partie : c'est qu'elle concerne un certain nombre de phénomènes qui ne sont pas faciles à démêler. Mais c'est aussi que, de la sorte, son extension dépasse celle que lui attribue la théorie de Goodman prise au pied de la lettre, laquelle ne concerne que l'exemple, sans aller jusqu'à penser le lien de ce dernier avec l'exemplarité. (Or, il me semble heuristique de traiter exemple et exemplarité dans une même forme de réflexivité pour au moins deux raisons, l'une conceptuelle et l'autre empirique. La raison conceptuelle est qu'il est important de voir - surtout dans une optique constructiviste comme celle de Goodman - qu'une chose peut être l'exemple d'un ensemble indéfini de qualités. En d'autres termes, rien, en soi, n'est un exemple de quoi que ce soit. Produire un exemple, exemplifier quelque chose, c'est toujours rentrer dans une axiologique qui valorise certaines propriétés, ce « quelque chose " précisément, au moment où l'on en institue l'exemple. Ainsi, l'échantillon de tissu qui exemplifie une couleur peut exemplifier aussi une texture, une longueur, une épaisseur, une brillance, etc.; il n'exemplifie que la couleur dans une situation donnée, où ce qui importe, c'est de disposer d'un terme de comparaison pour juger des couleurs des tissus. Produire un exemple, c'est constituer quelque chose en parangon. Car pour prendre la question a contrario : que pourrait bien être un exemple-non-exemplaire? Les raisons pour penser l'exemple-exemplaire ne sont pas seulement conceptuelles, mais aussi empiriques : en particulier, ici, il semble important de pouvoir rendre compte d'une très grande variété de phénomènes sémiotiques qui ont accompagné pratiquement toute l'histoire de l'art : les œuvres de toute sorte qui ont fonctionné comme exempla. Car depuis l'Antiquité à nos jours, s'il est peut-être une chose qui reste dans la sémiotique des productions artistiques, c'est précisément le regard éthique sur ces dernières - que celui-ci soit affirmé, nié ou interrogé : il s'agit de considérer ou de refuser expressément de

44. Deleuze (1981, notamment chapp. XII « Le diagramme » et XIII « L'analogie »).

45. Cf. Goodman (1968, chap. II « La sonorité des images » et 1978, passim). 
considérer les œuvres comme des modèles de comportement, à savoir des exemplifications, des incarnations, et des cas exemplaires, des schématisations suggestives, des manières de voir, de penser, d'agir ${ }^{46}$ ).

Pour commencer, d'un point de vue sémiotique, il semble que la chose la plus générique qu'on puisse dire d'une œuvre, c'est qu'elle exemplifie notre perception et compréhension par ce qu'elle nous fait percevoir et comprendre. Ainsi un film, quoi qu'il nous fasse percevoir, il nous fait percevoir aussi que nous percevons et la manière dont nous le faisons, ce que nous sommes en train de voir et entendre, ce que nous pouvons, savons, voulons voir et entendre. Certes, cela se passe à des degrés d'intensité variable, selon le film et la situation. Mais il est clair que, selon un certain point de vue, le sens d'un film est expérimenter que nous avons mal vu, que des choses importantes nous ont échappé : c'est ce qui se passe, typiquement, dans le film policier; ou encore que nous aimons entendre certains sons : c'est le sens générique de la comédie musicale; ou que nous nous dégoûtons de voir certaines images : c'est le sens générique du film d'épouvante. Tous ces phénomènes, dont personne n'ignore l'importance, sont bien réflexifs : nous expérimentons l'expérience, nous regardons le regard, nous percevons la perception. Et ce procès suiréférentiel exemplaire du film est bien partie de son sens : de son sens en tant que film d'un certain genre, par exemple; et il est même parmi les raisons de la jouissance que le film nous procure : voir des visions horribles pour le film d'épouvante, écouter des écoutes ravissantes pour la comédie musicale, etc. ${ }^{47}$

Qu'une œuvre fonctionne comme exemplum de quelque chose qu'elle possède, qu'elle soit exemplaire, cela veut dire que, après son expérience, nous expérimentons les autres œuvres et le monde en général d'après cette dernière. C’est là, encore une fois, une vérité commune dont il ne faut que tirer toutes les conséquences : après l'observation de certains tableaux, l'écoute d'une certaine musique, la lecture de certains romans, nous voyons les choses du monde différemment. C'est, précisément, ce qui se passe lorsqu'on dit, d'un bleu, qu'il est "Klein »; de certaines masses sonores, qu'elles sont "wagnériennes "; de certaines situations, qu'elles sont «felliniennes». C'est que les toiles de Klein ont exemplifié, notoirement, un bleu; les opéras de Wagner, une texture symphonique nouvelle; les films de Fellini, des microcosmes particuliers. Selon ce même procès sémiotique d'exemplification, nous pouvons, grâce à la littérature, voir et comprendre les choses du monde comme étant "dantesques », "pantagruéliques ", "donquichottesques », "sadiques ",

46. Sur cette question du regard éthique qui accompagne l'histoire de l'art, on se rapportera à l'étude de Talon-Hugon (2009; sur « la grande tradition de l'exemplum », le fait que « les arts de la représentation fournissent ainsi des exemples au double sens du mot: des exemplifications et des cas exemplaires", à la fois des « incarnations " et des "schèmes ", cf. notamment pp. 33-36, souligné dans le texte, ainsi que pp. 55-56 et 71-89).

47. C'est sans doute dans une telle optique, encore plus que dans l'optique de la figuralité, qu'on peut relire avec profit les écrits suggestifs de Schefer (1980 et le recueil 1999) sur le cinéma, qui constituent, historiquement, le premier dépassement de la sémiotique du cinéma et de l'image de dérivation structurale. 
« beylistes », « kafkaïennes », " ubuesques ». Or, le « fellinien » ou le « sadique » ne sont pas ce que les films de Fellini ou les romans de Sade racontent, décrivent, figurent du monde, mais plutôt le contraire : c'est ce que le monde possède de ces œuvres. En d'autres termes, les œuvres se présentent comme autant d'échantillonnages du monde - le monde qu'elles-mêmes contribuent à mettre en forme et construire.

Mais pourquoi un tel procès d'exemplification a-t-il lieu plus à travers l'histoire d'un roman qu'à travers la chronique d'un article de journal; plus à travers la performance des acteurs sur scène qu'à travers ce que notre mère fait à la maison? Nous avons déjà évoqué la réponse : parce que la réflexivité exemplaire est constitutive de la sémiotique de l'art et du spectacle. Ce ne sont pas les textes ou les faits sémiotiques en soi qui posséderaient une vertu exemplaire; c'est plutôt le fait même qu'un texte fasse sens en tant qu'œuvre littéraire, une action en tant que spectacle qui rend le texte ou l'action exemplaires. C'est aux œuvres d'art et aux spectacles qu'on demande surtout de modéliser le monde - même si on aurait pu le demander aux chroniques locales ou aux gestes de notre mère (mais il doit y avoir des gens qui, effectivement, leur demandent $)^{48}$. Cela explique ce phénomène si curieux et puissant pour lequel les œuvres et les spectacles, même s'ils parlent de choses lointaines, peuvent nous toucher plus profondément de ce qui représente des choses plus proches de nous. Cela explique pourquoi, par exemple, les films qui dépeignent la misère de l'Italie de l'après-guerre, tels Le voleur de bicyclettes ou Umberto $D$. de Vittorio De Sica, sont plus bouleversants que le fait divers qui concerne notre quartier aujourd'hui : c'est n'est pas ce qui est dit, décrit, figuré dans le film qui compte au final, mais ce qui par là est montré, modélisé, exemplifié; en l'occurrence, quelque chose comme la misère et la dignité humaines, l'injustice et la lutte pour la vie.

Certes, la "lutte pour la vie » chez De Sica est bien quelque chose de très général, tout comme le sont le "fellinien", le "sadique» ou l'« hamlétique». Oui, la réflexivité exemplaire, ou exemplification, fonctionne par un procès de grande généralisation; elle est donc le complémentaire de la réflexivité figurale, ou diagrammaticalisation, qui fonctionne par un procès de grande singularisation, de qualification bien vague. Il s'agit de deux procès imprécis, qui n'indiquent, n'assertent, rien de particulier, mais ouvrent un éventail de choses variables. En effet, comme l'a bien expliqué Peirce, il y a deux pôles opposés de l'imprécision : l'un, c'est le vague, qui concerne les qualités des phénomènes (nous dirons : leur figuralité); l'autre, c'est le général, qui concerne l'extension des phénomènes (nous dirons : leur exemplarité ${ }^{49}$. Dans le premier cas, où ce qui compte est la qualité de

48. Pour une argumentation plus développée sur la nature exemplifiante du spectacle (d'ailleurs non seulement le spectacle théâtral, mais aussi le spectacle sportif, le spectacle des procès judiciaires, etc.) : cf. encore Tore (2011).

49. Pour préciser la différence entre le vague de la qualité et le général de l'extension, les critères de Peirce (1905, p. 112 tr. fr.) sont les suivants : dans le vague, il y a la place pour la contradiction : 
quelque chose, le sens est pour ainsi dire interne à la chose même, il est quelque chose de singulier im-pliqué (c'est ce que le diagramme montre). Dans le deuxième cas, où ce qui compte est l'extension, la généralité d'une chose, le sens est en quelque sorte externe, ex-pliqué (c'est ce que l'exemple déploie) ${ }^{50}$.

On peut formuler la complémentarité de ces deux réflexivités en d'autres termes. Dans les deux cas, il est question d'une modélisation, à savoir de l'expression instructive d'une certaine variabilité sémiotique. Or, dans le premier cas, dans la réflexivité figurale ou diagrammaticalisation, la variabilité sémiotique est interne : il s'agit précisément d'une auto-modélisation, d'une auto-réflexivité; dans le second cas, dans la réflexivité exemplaire ou exemplification, la variabilité est externe : il s'agit d'une modélisation, ou d'une réflexivité qui est performative.

Cette différence s'avère utile pour distinguer les phénomènes réflexifs en général, non seulement les phénomènes de réflexivité globale. Les phénomènes de réflexivité diffuse ou ponctuelle peuvent être distingués sur un deuxième axe, où l'on aurait une réflexivité tournée vers l'extérieur, et donc généralisante, ou une réflexivité tournée vers leur intérieur, et donc singularisante. Et ce n'est que dans ce dernier cas qu'on parlera d'autoréflexivité, alors que pour l'autre on parlera de réflexivité simple.

\subsection{Deuxième axe, les orientations : réflexivité externe/interne, ou réflexivité simple/autoréflexivité}

Un des nombreux malentendus auxquels il faut faire face, dans la littérature non systématique et lacunaire sur la réflexivité, porte sur l'idée que "réflexif » et « autoréflexif» sont des synonymes. Je terminerai cette cartographie des phénomènes réflexifs en illustrant la différence très importante entre les deux. Pour commencer, songeons aux cas, bien fréquents, où dans un roman on parle d'un romancier, dans un film on voit un plateau de tournage. Nous pouvons nous demander : est-il question alors d'une mise en abyme? Par exemple, le romancier figuré dans le roman, est-il en train d'écrire le roman que nous sommes en train de lire, ou bien s'agit-il simplement d'un personnage dont le métier est d'être écrivain? La représentation du plateau de tournage dans un film parle-t-elle du film lui-même, ou bien d'un autre film, voire du monde du cinéma en général? Ces alternatives suggèrent que la réflexivité, ce n'est pas toujours de l'autoréflexivité. La réflexivité non autoréflexive est le simple fait que, par exemple, un roman parle de l'écriture, un film parle de tournages, une œuvre d'art parle du monde de l'art; ou aussi, comme nous venons de l'illustrer, qu'un film de Fellini parle du monde «fellinien », à savoir clownesque, onirique, grotesque, etc. Cette réflexivité

ainsi un animal vu vaguement peut être un mâle ou une femelle; dans le général, il y a la place pour le tiers exclu : ainsi « un triangle en général n'est ni isocèle ni équilatéral; un triangle en général n'est pas non plus scalène ».

50. Il faut savoir que, dans une autre perspective que la nôtre, on a aussi soutenu la généralité du diagramme (cf. Dondero, 2013). 
simple est donc orientée vers l'extérieur; elle est plus ou moins performative. En effet, lorsqu'une œuvre d'art parle du monde de l'art, elle change, plus ou moins puissamment, le monde de l'art auquel elle-même appartient; lorsque le film de Fellini est «fellinien", il change la manière de voir le monde qu'il a lui-même contribué à faire connaître. À cet égard, l'œuvre d'art, le film de Fellini constituent des exempla.

Tout cela est le contraire exact de l'autoréflexivité. La méprise peut-être plus commune sur la réflexivité dans les études de l'image et du cinéma consiste à considérer que toute figuration du monde de l'art dans une œuvre d'art, toute apparition de miroirs, de fenêtres et de n'importe quel cadre dans le cadre de l'image suffisent à produire le vertige de la mise en abyme. Or, dans la plupart de cas, il s'agit de réflexivité simple. Car ce n'est pas la figure ou le procédé en soi qui définit le sens de la réflexivité, mais le sens de l'œuvre, du texte, de l'image, du spectacle. Un cadre dans le cadre peut aussi faire sens comme autoréflexif, tout comme n'importe quel autre élément d'une œuvre. La condition, c'est que son sens soit de questionner le sens de l'œuvre même, modéliser l'intérieur de cette dernière ${ }^{51}$. Ainsi, dans Citizen Kane, le fameux puzzle qui apparaît dans les mains de Susan Kane vers la fin du film est bien autoréflexif, à savoir une mise en abyme du film entier, puisqu'à ce moment du film il est très clair que ce dernier est totalement construit comme un puzzle, dont on essaie de recomposer les morceaux; mais, bien sûr, ce n'est pas pour autant que tout puzzle qui apparaîtra dans n'importe quel film rendra ce dernier autoréflexif ${ }^{52}$.

Le puzzle de Citizen Kane, la mise en abyme en général constituent clairement une réflexivité interne (une autoréflexivité) localisée, ponctuelle. Le raisonnement des paraboles évangéliques, la logique de la sensation dans l'œuvre de Bacon selon Deleuze, la pensée figurale de l'interpénétration des mondes dans Lynch selon Basso constituent autant de réflexivités internes (des autoréflexivités) globales. Nous sommes ici en train de projeter l'autoréflexivité, qui est une question d'orientation, sur l'axe des modes de la manifestation. De la sorte, nous pouvons voir qu'entre les cas, clairs et extrêmes, de la mise en abyme localisable, d'une part, et de la diagrammaticalisation globale, d'autre part, il est beaucoup de cas mixtes très intéressants et bien fréquents; des cas de réflexivité à la manifestation dynamique : locale, diffuse et globale; des cas où il est difficile de statuer d'une manière définitive la limite entre une marque d'autoréflexivité et une autre, un moment explicite d'autoréflexivité et un autre; des cas, finalement, où l'œuvre en

51. Encore une fois, ces questions ont été étudiées de manière très analytique dans la cartographie essentielle de Metz (1991).

52. Pour revenir, à partir de cet exemple, sur la question du «méta » : on peut dire que le puzzle dans Citizen Kane est autoréflexif mais pas métacinématographique : le puzzle continue bien d'être élément filmique, partie de la figuration, avec Susan Kane qui y joue ennuyée, ou le journaliste Thompson qui le montre à tout le monde à la fin de son enquête ratée; et en même temps le puzzle est aussi une figuration opaque, qui sort des mains de Susan ou de Thompson pour être hissée à présence instructive de tout le film, monstration de son sens. 
question devient une sorte de mise en abyme totale d'elle-même. Que l'on songe à un film comme Huit et demi de Fellini. Il raconte l'histoire d'un film qu'on doit tourner : il s'agit donc un film réflexif; mais ce film qu'on doit tourner et qu'on ne cesse de mettre en scène, finalement, s'avère être Huit et demi lui-même : le film est donc autoréflexif, et de manière globale. Ainsi, finalement, les nombreux moments de film dans le film peuvent fonctionner comme autant de traces qui explicitent et alimentent le sens du film tout entier - lequel devient alors une sorte de diagramme de ses possibilités d'être film ${ }^{53}$. (Mais un tel cas de figure doit être dissimilé, par exemple, de La nuit américaine, le film de Truffaut : un film qui parle d'un tournage et réfléchit simplement le monde du cinéma, sans qu'il n'y ait d'enchevêtrements vertigineux entre, d'une part, le film énonciateur, le film qui se montre, se présente au spectateur et, d'autre part, le film énoncé, le film qu'est raconté, représenté.) À son tour, Huit et demi ne doit pas faire penser que, dans les cas mixtes, la réflexivité simple doive forcément précéder l'autoréflexivité : Le mépris de Godard commence par une impressionnante mise en abyme (la vision d'un tournage embrayé et même assumé par la voix de Godard, qui liste le cast and credits du film), il récupère ensuite la figuration dans le mode évident de la réflexivité simple (un film sur une histoire de tournage de film), il laisse finalement planer le doute sur le sens autoréflexif de sa réflexion sur le monde du cinéma... ${ }^{54}$

Nous pouvons revenir, pour conclure, sur la différence de sens entre réflexivité simple, tournée vers l'extérieur, et autoréflexivité, tournée vers l'intérieur. Le sens de la réflexivité simple d'un objet ou d'un événement, c'est de généraliser la sémiotique de l'objet ou de l'événement en question. Ainsi, un cadre dans le cadre montre et valorise le cadrage en tant que tel, en tant que principe de construction des images. Ainsi, une citation dans un texte apparente ce dernier au texte cité, et donc montre et alimente le principe d'une «transtextualité » — une littérature ou un art « au second degré », comme Genette a pu l'illustrer ${ }^{55}$. Mais que l'on songe, par exemple, aux œuvres situationnistes : des textes qui ne sont faits, clairement, que de citations d'autres textes; il y a alors quelque chose de plus qui se produit : non seulement le texte est apparenté à une myriade de textes, son sens devient surtout un questionnement sur sa propre construction en tant que texte. Le sens n'est plus tellement généralisant, orienté vers d'autres textes, que singularisant,

53. Pour une analyse : cf. Metz (1966).

54. La plus grande limite de l'étude de Dällenbach (1977), devenue référence essentielle dans la littérature sur la mise en abyme, est d'avoir voulu restreindre la conception de cette dernière : selon Dällenbach, la mise en abyme, pour avoir raison d'être, devrait être marquée formellement et même signalée diégétiquement; autrement dit, elle devrait comporter une structure d'enchâssement et un récit intradiégétique ou métadiégétique. Ce serait alors exclure de l'étude de la mise en abyme la plupart des exemples que nous avons discutés et, surtout, avec eux, l'extrême complexité de l'autoréfléxivité que nous avons défendue dans ces pages (cf. aussi la critique à Dällenbach dans Vouilloux, 2004, chap. IV, où l'on montre par ailleurs que l'enchâssement est loin d'être la seule possibilité d'enclave, d'abyme).

55. Genette (1982). 
orienté vers la construction même du texte en question. Le sens n'est plus tellement réflexif, il est carrément autoréflexif.

C'est dire que, dans la réflexivité simple, on alimente l'intertextualité (ou l'interartisticité) en général, et par là on valorise, et même on glorifie ce monde même fait de textes (ou d'œuvres d'art). Par contre, dans l'autoréflexivité, on semble plutôt miner la textualité ou la composition artistique en tant que telles, ou en tout cas on en limite le fonctionnement. Ainsi, Huit et demi semble presque se dissoudre en tant que film; le puzzle de Citizen Kane particularise et littéralement décompose le film même. Dans les termes de Christian Metz, dans la réflexivité simple du cadre dans le cadre ou du film dans le film, il y a un «doublement» du foyer énonciatif; alors que dans l'autoréflexivité de la mise en abyme, qui semble presque faire imploser l'image ou le film, il y a un «dédoublement» du foyer de l'énonciation ${ }^{56}$.

\section{Références bibliographiques}

Aubral, François et Chateau, Dominique (éds, 1999), Figure, figural, Paris, L’Harmattan.

Aumont, Jacques (1996), À quoi pensent les films?, Paris, Séguier.

Austin, John L. (1962), How to Do Things with Words, Oxford University Press, posthume; tr. fr. Quand dire, c'est faire, Paris, Seuil, 1970.

Authier-Revuz, Jacqueline (1995), Ces mots qui ne vont pas de soi. Boucles réflexives et non-coïncidences $d u$ dire, Paris, Larousse.

BaLLy, Charles (1932), Linguistique générale et linguistique française, Paris, Leroux; nouv. éd. Bern, Francke, 1965.

Basso Fossali, Pierluigi (2006), Interpretazione tra mondi. Il pensiero figurale di David Lynch, Pisa, ETS; $2^{e}$ éd. 2008.

Benveniste, Émile (1958), « De la subjectivité dans le langage », Journal de Psychologie, 55, pp. 257-265; repris dans Problèmes de linguistique générale, 1, Paris, Gallimard, 1966, chap. XXI.

- (1963), «La philosophie analytique et le langage», Les Études philosophiques, 1; repris dans Problèmes de linguistique générale, 1, Paris, Gallimard, 1966, chap. XXII.

Beyaert-Geslin, Anne et Fontanille, Jacques (éds, 2003), «Modèles sémiotiques et textualité », numéro de Modèles linguistiques, 47.

Chanady, Amaryll (1988), « Une métacritique de la métalittérature : Quelques considérations théoriques », Études françaises, 23/3, pp. 135-145.

DäLlenbaCh, Lucien (1977), Le récit spéculaire. Essai sur la mise en abyme, Paris, Seuil.

Deleuze, Gilles (1981), Francis Bacon. Logique de la sensation, Paris, La Différence; $2^{\mathrm{e}}$ éd. 1984.

56. Toujours Metz (1991). 
Didi-Huberman, Georges (1990), Devant l'image. Question posée aux fins d'une histoire de l'art, Paris, Minuit.

Dondero, Maria Giulia (2014), "La totalité en science et en art », in Beyaert-Geslin, Anne et Dondero, Maria Giulia (éds), Arts et sciences. Approches sémiotiques et philosophiques des images, Presses Universitaires de Liège, pp. 123-136.

Ducrot, Oswald (1979), "Les lois du discours », Langue française, 42, pp. 21-33; repris dans Le dire et le dit, Paris, Minuit, 1984, chap. V.

- (1984), Le dire et le dit, Paris, Minuit.

- (1986), «Charles Bally et la pragmatique », Cahiers Ferdinand de Saussure, 40, 13-37; repris comme "Énonciation et polyphonie chez Charles Bally», in Logique, structure, énonciation. Lectures sur le langage, Paris, Minuit, 1989, chap. VII.

Fontanille, Jacques (2008), Pratiques sémiotiques, Paris, PUF.

Fontanille, Jacques et Zilberberg, Claude (1998), Tension et signification, Liège, Mardaga.

Fraisse, Luc (2011), «L'autoréflexivité en pratique », Poétique, 152, pp. 155-170.

Freud, Sigmund (1899), Die Traumdeutung, Leipzig-Wien, Deuticke ; $8^{\mathrm{e}}$ éd. 1930; tr. fr. L'interprétation des rêves, Paris, PUF, 1926; 2e éd. 1967.

Garfinkel, Harold (1967), Studies in Ethnomethodology, Engelwood Cliffs, NJ, PrenticeHall; tr. fr. Recherches en ethnométhodologie, Paris, PUF, 2007.

Garfinkel, Harold et Sacks, Harvey (1970), «On Formal Structures of Practical Actions », in J. McKinney et E. Tiryakian (éds), Theoretical Sociology. Perspectives and Developments, Appelton Century Crofts, pp. 338-366; tr. fr. in H. Garfinkel, Recherches en ethnométhodologie, Paris, PUF, 2007, " Appendice ».

Genin, Cristophe (1998), Réflexions de l'art. Essai sur l'autoréférence en art, Paris, Kimé.

Geertz, Clifford (1973), The Interpretation of Cultures. Selected Essays, New York, Basic Books.

- (1983), Local Knowledge. Further Essays in Interpretative Anthropology, New York, Basic Books; tr. fr. Savoir local, savoir global. Les lieux du savoir, Paris, PUF, 1986.

Genette, Gérard (1982), Palimpsestes. La littérature au second degré, Paris, Seuil.

- (1987), Seuils, Paris, Seuil.

- (1991), Fiction et diction, Paris, Seuil.

Goffman, Erving (1974), Frame Analysis. An Essay of the Organization of Experience, London, Harper \& Row; tr. fr. Les cadres de l'expérience, Paris, Minuit, 1991.

- (1977), "The Arrangement between the Sexes ", Theory and Society, 4/3, 301-331; tr. fr. L'arrangement des sexes, Paris, La Dispute, 2002.

Goodman, Nelson (1968), Languages of Art. An Approach to a Theory of Symbols, London, Bobbs-Merril; tr. fr. Les langages de l'art. Une approche de la théorie des symboles, Nîmes, J. Chambon, 1990. 
- (1978), Ways of Worldmaking, Indianapolis, Hackett; tr. fr. Manières de faire des mondes, Nîmes, J. Chambon, 1992.

Greimas, Algirdas J. et Courtés, Joseph (1979), Sémiotique. Dictionnaire raisonné de la théorie du langage, Paris, Hachette.

Hjelmslev, Louis (1943), Omkring Sprogteoriens Grundloeggelse, København, Munksgaard; tr. fr. Prolégomènes à une théorie du langage, Paris, Minuit, 1971.

НumвоLdт, Wilhelm von (1836), Über die Verschiendenheit des menschlichen Sprachbaues und ihren Einfluß auf die geistige Entwicklung des Menschengeschlechts, Berlin, Dümmler; tr. fr. in Introduction à l'œuvre sur le kawi. Et autres essais, Paris, Seuil, 1974.

Kerbrat-Orecchioni, Catherine (1980), L'Énonciation. De la subjectivité dans le langage, Paris, Armand Colin; $2^{\mathrm{e}}$ éd. 2002.

- (1986), L’implicite, Paris, Armand Colin.

Kleiber, Georges (1986), « Déictiques, embrayeurs, 'token-réflexives', symboles indexicaux, etc. : Comment les définir?", L’information grammaticale, 30, pp. 3-22.

Luhmann, Niklas (1984), Soziale Systeme. Grundriß einer allgemeiner Theorie, Frankfurt, Suhrkamp; tr. fr. Systèmes sociaux, Québec, Presses de l'Université de Laval, 2010.

LyotARd, Jean-François (1971), Discours, figure, Paris, Klincksieck.

Maingueneau, Dominique (1995), «L'énonciation philosophique comme institution discursive ", Langages, 119, pp. 40-62.

Marin, Louis (1977), Détruire la peinture, Paris, Galilée; 2éd. Flammarion, 1997.

- (1988), "Mimésis et description", Word and Image - A Journal of Verbal/Visual Enquiry, 4/1, pp. 25-36; repris dans De la représentation, Paris, Gallimard/Seuil, 1994, chap. 15.

- (1989), Opacité de la peinture. Essais sur la représentation au Quattrocento, Paris, Usher; $2^{\mathrm{e}}$ éd. Éditions de l'EHESS, 2006.

- (1994), De la représentation, Paris, Gallimard/Seuil, recueil posthume.

Merleau-Ponty, Maurice (1945), Phénoménologie de la perception, Paris, Gallimard.

Metz, Christian (1966), «La construction 'en abyme' dans Huit et demi, de Fellini », Revue d'esthétique, XIX/1, pp. 96-101; repris dans Essais sur la signification au cinéma, Paris, Klincksieck, 1968, chap. 9.; $2^{\mathrm{e}}$ éd. 1971.

- (1977), Le signifiant imaginaire. Psychanalyse et cinéma, Paris, UGE; 2 éd. Christian Bourgeois, 1984.

- (1991), L'énonciation impersonnelle. Ou le site du film, Paris, Klincksieck.

Peirce, Charles S. (1893), "Short Logic», inédit; in Collected Papers, 2.282, 2.286-91, 2.295-6, 2.435-44, 7.555-8. 
- (1895), «That Categorical and Hypothetical Propositions Are One in Essence, with Some Connected Matters ", inédit; in Collected Papers, 2.278-80.

- (1903), "Syllabus (of Certains Topics of Logic) », inédit; in Collected Papers, 1.180-202, 2.219-26, 2.274-7, 2.283-4, 2.292-4, 2.309-31, 2.640-2, 3.571-608, 4.394-417.

- (1905), «Pragmaticisme, Prag. [4] », inédit; in Collected Papers, 5.502-37; tr. fr. "Le pragmaticisme, Prag [4] », Euvres II. Pragmatisme et sciences normatives, Paris, Cerf, 2003, pp. 107-133.

- (1906), « Prolegomena to an Apology for Pragmaticism », The Monist, 16, pp. 492-546; in Collected Papers, 4.530-72.

- (1931-58), Collected Papers, Cambridge (Mass.), Harvard University Press, posthume.

RANCière, Jacques (2004), Malaise dans l'esthétique, Paris, Galilée.

Rastier, François (2001), Arts et sciences du texte, Paris, PUF.

RÉCAnati, François (1979), La transparence et l'énonciation. Pour introduire à la pragmatique, Paris, Seuil.

Schefer, Jean Louis (1980), L’homme ordinaire du cinéma, Paris, Cahiers du cinémaGallimard.

— (1999), Images mobiles. Récits, visages, flocons, Paris, P.O.L., recueil 1978-1998.

Talon-Hugon, Carole (2009), Morales de l'art, Paris, PUF.

Tiercelin, Claudine (1993), La pensée-signe. Études sur C.S. Peirce, Nîmes, J. Chambon.

Tore, Gian Maria (2008), «Compte rendu de Pierluigi Basso Fossali, Interpretazione tra mondi. Il pensiero figurale di David Lynch, Pisa, ETS, 2006 », Actes Sémiotiques, en ligne : http://epublications.unilim.fr/revues/as/2237 (consulté le 17/9/2013).

- (2011), "Pour une sémiologie du spectaculaire : définitions et questions », Actes sémiotiques, en ligne : http://epublications.unilim.fr/revues/as/1914 (consulté le 17/9/2013).

VANCHeri, Luc (2011), Les pensées figurales de l'image, Paris, Armand Colin.

Vouilloux, Bernard (2004), L'œuvre en souffrance. Entre poétique et esthétique, Paris, Belin.

WESSLER, Eric (2009), La littérature face à elle-même. L'écriture spéculaire de Samuel Beckett, Amsterdam-New York, Rodopi. 